P80 GENITOURINARY MEDICINE PHYSICIANS NEED FURTHER TRAINING IN THE MANAGEMENT OF HSV IN LATE PREGNANCY

doi:10.1136/sextrans-2012-050601c.80

${ }^{1} \mathrm{C}$ Fleury, ${ }^{*} \mathrm{E}$ Clarke, ${ }^{1} \mathrm{~A}$ Baker, ${ }^{3} \mathrm{~J}$ Green, ${ }^{2} \mathrm{E}$ Foley, ${ }^{4} \mathrm{R}$ Patel. ${ }^{1}$ University of Southampton, Southampton, UK; ${ }^{2}$ Royal South Hants Hospital, Solent NHS Trust, Southampton, UK; ${ }^{3}$ St. Mary's Hospital, Madison, Wisconsin, USA; ${ }^{4}$ University of Southampton \& Royal South Hants Hospital, Southampton, UK

Background Transmission rates of neonatal herpes simplex virus (HSV) infection varies from $30 \%$ to $50 \%$ if shedding with primary infection during the third trimester of pregnancy, to $<3 \%$ with active recurrent genital infection. Despite the low transmission risk of recurrent HSV in pregnancy, there remains confusion regarding appropriate management.

Aim To investigate the level of knowledge of BASHH guidelines regarding the management of $\mathrm{GH}$ in the third trimester of pregnancy amongst BASHH conference attendees.

Methods Attendees of two BASHH conferences completed a questionnaire consisting of case-scenarios regarding appropriate management of pregnant women and their partners with genital herpes infection. The first case-scenario was designed to assess familiarity with accepted guidance. The second was used to assess whether physicians followed RCOG or BASHH guidelines.

Results 94 attendees completed the survey. In line with current guidelines, $81(74 \%)$ answered that primary HSV infection at term was an indication for Caesarian section (CS), and 37 (46\%) of these 81 responders stated this CS should be carried out at 38 weeks gestation. Of these 37 responders, when questioned concerning recurrent genital infection at term, $27(73 \%)$ believed that lesions present at delivery would necessitate a CS, and 35 (95\%) felt that vaginal delivery was appropriate in the absence of lesions at delivery. Although there is specific guidance for the limited place of invasive techniques at term only $17 \%$ would advise avoidance of fetal scalp electrodes and artificial membrane rupture.

Conclusion No-one demonstrated complete familiarity with the BASHH guidelines surrounding appropriate management of HSV. Of most concern is the limited awareness of the management need of CS in third trimester first episode disease. Further training of GUM physicians is required in order to ensure practice is compatible with the best available advice.

\section{P81 POINT (YOUNG PERSON'S) CLINIC AUDIT}

doi:10.1136/sextrans-2012-050601c.81

${ }^{1} S$ Y Chan, * 2J Zhou, ${ }^{2}$ S Rashbrook, ${ }^{2 B}$ Dragovic. 'St Georges Healthcare NHS Trust, London, UK; ${ }^{2}$ Queen Mary's Hospital, London, UK

Background Young people attending sexual health clinics should receive an adequate standard of care, according to BASHH clinical effectiveness group guidance.

Aims/Objectives To carry out an audit of clients attending the Point clinic - a sexual health clinic for clients aged $\leq 18$. To describe client characteristics and compare practice against BASHH guidelines.

Methods We carried out a retrospective case notes review of all clients attending the Point clinic from 1 November 2009 to 30 April 2010. Patients were only counted once (new/rebook). Only patients having sexually transmitted infection (STI) screens were included. Information was collected on demographics, risk assessment, STI screening, HIV testing and contraception.

Results 123 clients attended. 51 did not have a screen. Reasons were: not sexually active, requesting contraception/condoms only, attending for results. Included in the audit were 72 patients who had STI screens. 53 out of $72(73.6 \%)$ were female with a mean age of 16.9. 10 out of $72(13.9 \%)$ were $<16$. Fraser competence was assessed in all $<16$ years old. Risk assessment (age of partner/ alcohol/drugs use/housing/mental health issues) was documented in $63 / 72(87.5 \%)$ and in all $<16$ year olds. Contraception was reviewed in $64 / 72(88.9 \%)$. Additional contraception advice was given to $36 / 72(50 \%)$. Of the 34 not given additional contraceptive advice, six were not using any contraception. HIV test was offered to $71 / 72$ and 35 accepted. 11/72 (15.3\%) had an STI, most common being Chlamydia trachomatis (6/11).

Conclusion We are managing patients in accordance with BASHH guidelines. All $<16$ year olds (and those 16-18 where indicated) had a risk assessment completed. Offer of HIV tests was very good. It was identified that improvements were required with regards to provision of additional contraceptive advice.

\section{Women and children}

\section{P82 WHAT DO PHASE 1 MICROBICIDE TRIAL PARTICIPANTS REALLY THINK? ARE THEY "ONLY IN IT FOR THE MONEY?"}

doi:10.1136/sextrans-2012-050601c.82

${ }^{1} \mathrm{G}$ Morris, ${ }^{*}{ }^{2} \mathrm{~S}$ Douglas, ${ }^{1} \mathrm{~S}$ Woodhall, ${ }^{1} \mathrm{C}$ Lacey. ${ }^{1}$ Hull-York Medical School, York, UK; ${ }^{2}$ York Hospital, York, UK

Background Early-phase trials require multiple visits and procedures, which can lead to difficulties with recruitment and compliance.

Aims To explore participants' experience of, and motivation for, taking part in a study of the safety and pharmacokinetics of an HIV microbicide.

Methods 28 women completed eight study visits including pelvic examinations, colposcopy, vaginal sampling (clinician performed and self-taken aspirate) and semi-structured, qualitative interviews. Additional requirements included daily vaginal gel use (12 doses) and sexual abstinence (16 days). Interviews were audio-recorded, transcribed, and analysed using a framework approach.

Results Although renumeration was given by $64 \%$ of women as the main or a contributing reason for participating, $71 \%$ also stated that they had an interest in HIV or saw the value in the research. $46 \%$ volunteered after hearing positive reports from other participants. Difficulties included speculum examinations, sampling, and fitting study visits and gel use into a normal routine. Problems with selfsampling were identified by 12 women, including discomfort and concern about obtaining an adequate sample. 10 participants reported a preference for self-sampling, 11 for clinician sampling. Compliance with gel use was high. Leakage was common but varied with position and depth of insertion. Four women found abstinence difficult. Being single, or discussing the study with partners before enrolment made abstinence easier.

Conclusion Qualitative interviews in early-phase trials capture useful additional information and help determine acceptability of procedures and products. Healthy subjects commonly have multiple non-exclusive reasons for participating in research. Understanding participants' motivation and experiences can aid recruitment and facilitate compliance with study requirements.

\section{P83 REVIEW OF THE FIRST ATTENDANCES IN UNDER- 16 YEAR OLDS IN A COMMUNITY BASED INTEGRATED SEXUAL HEALTH SERVICE}

doi:10.1136/sextrans-2012-050601c.83

N Pal, ${ }^{*} \mathrm{~J}$ Ashby. Berkshire Healthcare Foundation Trust, Bracknell, UK

Aim To analyse sexual and risk behaviour in under 16 year olds newly attending our services. 
Methods Computer databases were used to identify the last 100 under 16 year olds who newly attended the sexual health services up until December 2011. Patient records were retrospectively reviewed and clinical, social, sexual and reproductive data were collated using excel.

Results Age of the attendees ranged from 13 to 15 years; 95\% were girls. $98 \%$ reported heterosexual orientation. Ethnicity and primary reason for attendance as shown in abstract P83 table 1. Fraser competence was documented as present in 100\% of attendees. Vulnerability factors were assessed in all attendees. Age at coitarche ranged from 9 years of age upwards. $94 \%$ were sexually active and $91 \%$ reported unprotected sexual intercourse. Other than condoms, no other forms of contraception were in use. Contraception was discussed with $100 \%$ of attendees and $47 \%$ commenced contraception at first visit. $6 \%$ had at least one previous unplanned pregnancy. $9 \%$ reported symptoms of a sexually transmitted infection and $10 \%$ of attendees were diagnosed with at least one sexually transmitted infection at the first visit. $6 \%$ reported non-consensual sex of which $2 / 6$ were reported to the Police. $45 \%$ reported partners over age $16.15 \%$ had social services or other agencies input.

\section{Abstract P83 Table 1 Ethnicity and primary reason for attendance}

\begin{tabular}{lc}
\hline & $\%$ of attendees \\
\hline Ethnicity & 81 \\
White UK & 7 \\
White other & 6 \\
Black African/Caribbean & 5 \\
Asian & 1 \\
Mixed & \\
Primary reason for attendance & 41 \\
Contraception planning & 28 \\
Emergency contraception & 19 \\
Sexual health screen & 6 \\
Sexual health advice & 4 \\
Termination of pregnancy & 2 \\
Sexual assault & \\
\hline
\end{tabular}

Conclusions High rates of underage sexual activity and unsafe sex were observed in this group of young people attending clinic for the first time, with significant amounts of sexually transmitted infections identified and unplanned pregnancies seen. This highlights a need for earlier interventions, as often sexual risk had already occurred prior to the young people attending clinic.

\section{P84 WHERE DO BABIES COME FROM? A MAN'S UNDERSTANDING OF WOMEN'S HEALTH}

doi:10.1136/sextrans-2012-050601c.84

M F Appleby,* F Allen, B Dowsing, H Rich, P Douglass. UCL Medical School, London, UK

Background As part of a student selected study component, five male fourth year medical students sought to examine young men's knowledge of women's reproductive health. The importance of raising awareness of women's health must not be underestimated. Education of men in basic women's health could result in a significant reduction in unwanted pregnancy, misuse of contraception and domestic violence.

Aim(s)/Objective(s) The research question was simple-was our ignorance representative of our peers, and specifically, in which areas was knowledge most lacking?

Methods 24 questions were chosen for simplicity, guided by the developers' own lack of knowledge. Respondents were male pre-clinical medical students $(n=41)$ aged $18-21$, with a smaller but representative female cohort $(n=12)$ to be used for comparison.

Results and Discussion Male respondents' overall mean grade $48.3 \%$ (SD 16.9\%) compared poorly to the female respondents' $70.8 \%$ (SD $10.9 \%)$, significant on between samples t-test $(p<0.01)$. Results for each category are represented in abstract P84 table 1. Male respondents scored poorly on contraceptive awareness. The majority believed $\mathrm{A} \& \mathrm{E}$ to be an appropriate place to access emergency contraception. Not one male was able to name a serious side effect of the COCP. Only $7 \%$ of respondents knew the age women should receive their first cervical smear, and less than half knew its purpose. The project was limited by the opportunity sample used - medical students are well educated but unworldly in many other ways and not representative of other men their age. However, this study outlined the key areas where male education needs to be improved in an effort to involve men in women's health and combat inequalities.

Abstract P84 Table 1 Correct male responses regarding female sexual health

\begin{tabular}{ll}
\hline Menstrual cycle & $60 \%$ \\
Contraception & $47 \%$ \\
Cervical Screening & $27 \%$ \\
Menopause & $64 \%$ \\
Anatomy & $49 \%$ \\
\hline
\end{tabular}

\section{P85 CONGENITAL SYPHILIS IN THE UK: RESULTS FROM ENHANCED SURVEILLANCE, 2010/11}

doi:10.1136/sextrans-2012-050601c.85

${ }^{1}$ I Simms, ${ }^{*}{ }^{2} \mathrm{P}$ Tookey, ${ }^{3} \mathrm{~B}$ Goh, ${ }^{4} \mathrm{H}$ G Lyall, ${ }^{1} \mathrm{~B}$ Evans, ${ }^{1} \mathrm{C}$ Ison. ${ }^{1}$ Health Protection Agency, London, UK; ${ }^{2}$ UCL Institute of Child Health, London, UK; ${ }^{3}$ St Bartholomew's Hospital, London, UK; ${ }^{4}$ St Mary's Hospital, London, UK

Background Since 2001, rates of congenitally transmissible syphilis have increased amongst reproductive age women. Concerns have been raised about the effectiveness of intervention which has been restricted by an absence of national surveillance data. In 2010, a 3 -year study was established to provide an evidence-base for control strategies.

Aim To explore the epidemiology of congenital syphilis.

Methods The surveillance dataset consisted of returns to the British Paediatric Surveillance Unit, diagnoses made in genitourinary medicine clinics (GUMCAD) and laboratory reports. Infants diagnosed with congenital syphilis under the age of 24 months were included (detailed methodology at: http://www.rcpch.ac.uk/).

Results Between 1 February 2010 and 1 November /2011, 11 cases (male $=9$, female $=2$ ) of congenital syphilis were confirmed, all reported from England. Most infants $(6 / 10)$ were born at over 37 weeks: median birth weight was $2910 \mathrm{~g}$ (range: $1340 \mathrm{~g}$ to $3690 \mathrm{~g}$ ). Clinical presentation varied from asymptomatic (1) to acute, including severe anaemia, hepatosplenomegaly, rhinitis, oedema, thrombocytopaenia and skeletal damage. Median maternal age at delivery was 19 (range: 17-28). Many of the infants' mothers were White and came from the UK and Eastern Europe. Where maternal stage of infection was recorded, 4/7 presented with primary and 3/7 with secondary syphilis. One infection diagnosed late in pregnancy was the result of re-infection acquired after effective screening and management in the first trimester. All diagnoses were managed according to BASHH Guidelines.

Conclusions Congenital syphilis presents a complex social, clinical and public health problem. Antenatal screening is largely effective in controlling congenital syphilis but delivering effective health care to women who are marginalised in society and at-risk of acquiring congenitally transmissible syphilis represents an emerging challenge. 